\title{
APPLICATION OF DECISION TREE ON COLLISION AVOIDANCE SYSTEM DESIGN AND VERIFICATION FOR QUADCOPTER
}

\author{
Chun-Wei Chen, Ping-Hsun Hsieh*, Wei-Hsiang Lai \\ Dept. of Aeronautics and Astronautics, National Cheng Kung University, No. 1, University Road, Tainan, Taiwan \\ *P46041518@ncku.edu.tw
}

KEY WORDS: Quadcopter, Decision Tree, Collision Avoidance, System Design, Flight Testing.

\begin{abstract}
:
The purpose of the research is to build a collision avoidance system with decision tree algorithm used for quadcopters. While the ultrasonic range finder judges the distance is in collision avoidance interval, the access will be replaced from operator to the system to control the altitude of the UAV. According to the former experiences on operating quadcopters, we can obtain the appropriate pitch angle. The UAS implement the following three motions to avoid collisions. Case1: initial slow avoidance stage, Case2: slow avoidance stage and Case3: Rapid avoidance stage. Then the training data of collision avoidance test will be transmitted to the ground station via wireless transmission module to further analysis. The entire decision tree algorithm of collision avoidance system, transmission data, and ground station have been verified in some flight tests. In the flight test, the quadcopter can implement avoidance motion in real-time and move away from obstacles steadily. In the avoidance area, the authority of the collision avoidance system is higher than the operator and implements the avoidance process. The quadcopter can successfully fly away from the obstacles in 1.92 meter per second and the minimum distance between the quadcopter and the obstacle is 1.05 meters.
\end{abstract}

\section{INTRODUCTION}

With the developing of technology, the unmanned aerial vehicles (UAVs) are widely used on civilization and military field such as photogrammetry, entertainment, climate detecting, pesticide sprayed, etc. However, there are many safe issues that UAVs might encounter such as striking buildings and flying to restricted areas. Operators are constrained by view in the indoor environment or beyond visual range flight and can cause unsafely control that might hurt people, destroy something. Therefore, the collision avoidance system becomes more important (Chee, et al., 2013, Tripathi, et al., 2014). Chong-Ren Xiao (Xiao, 2009) developed collision avoidance algorithm based on fuzzy control and decision tree to make automatic mobile vehicle collision avoidance with merely 9 centimeter per second; Bing-SyunWu (Wu, 2015) integrated fuzzy controller to the quadcopter and verified the possibility of collision avoidance algorithm based on fuzzy control theory. The aim of the research is to integrate the ultrasonic range finder and decision tree algorithm (Sun, et al., 2017, Westreich, et al., 2010). Pixhawk flight control board is equipped on the quadcopter as the platform. The goal is to detect the front obstacles and to do corresponding avoidance motion in real time without extra equipment such as Arduino. While the ultrasonic range finder judges the distance is in collision avoidance interval, the access will be replaced from operator to the system to control the altitude of the UAV. The setting of pitch angle is according to the experience of the operator to implement following case: case1: initial slow avoidance stage, case2: slow avoidance stage. Case3: Rapid avoidance stage. The training data are transmitted by wireless transmission module to the ground data station in real time and plotted in graph to further discussion.

\section{EXPERIENTAL EQUIPMENT}

As shown in Figure 1 to Figure 4, the equipment of research including Pixhawk flight control board, the ultrasonic range finder, wireless transmission module (slave), and the quadcopter. The core of system structure is Pixhawk flight control board, the ultrasonic range finder utilizes $I^{2} C$ communication. Using Serial port connects wireless transmission module (slave). The flight control board receives the information such as flight attitude, remote control input signal, distance value received by the ultrasonic range finder, etc. The training data are transmitted by wireless transmission module to the ground data station, then using PLX-DAQ to print data real time on Excel to analyse flight data and plot it. The system structure is illustrated in Figure 5.

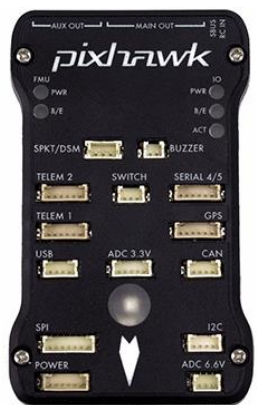

Figure 1 Pixhawk flight control board

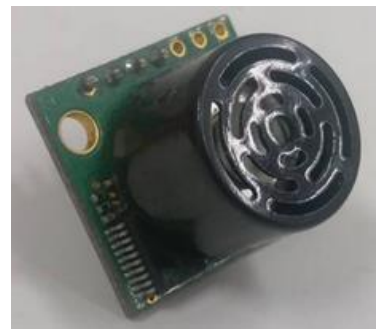

Figure 2 MB1202 ultrasonic range finder

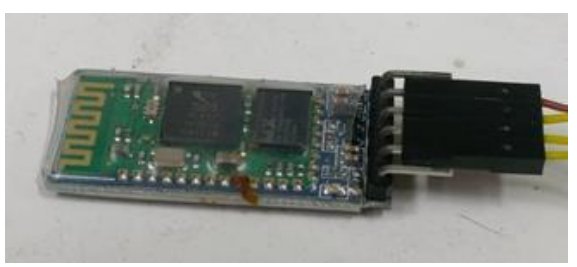

Figure 3 HC-05wireless blue tooth module 


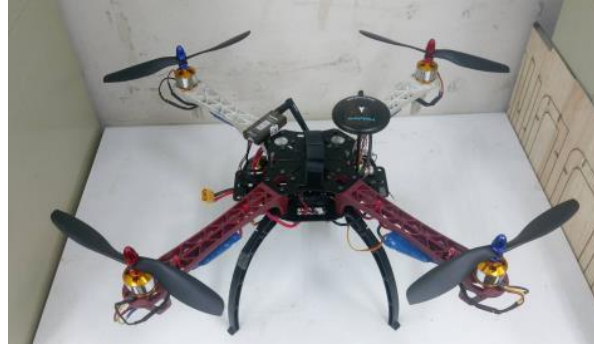

Figure 4 Quadcopter

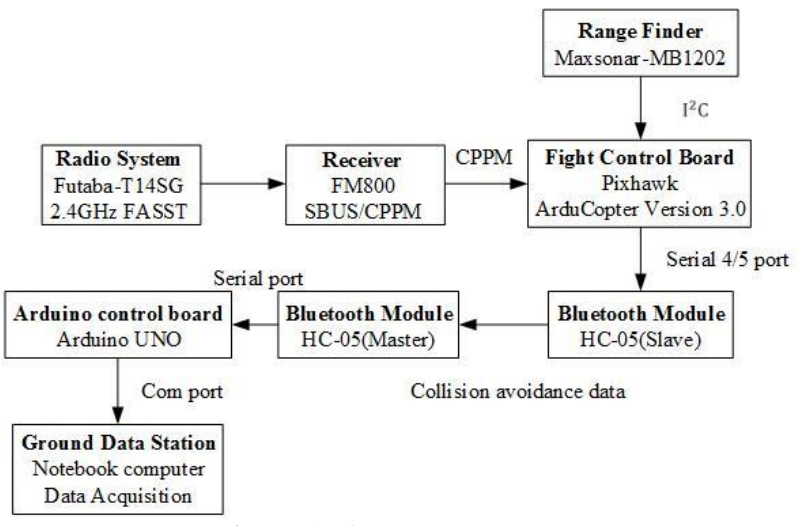

Figure 5 The system structure

\section{CONTROLLER DESIGN}

\subsection{Input Linear Operation}

Operating the handle makes the quadcopter move. The operator does not calculate angle and angular velocity directly. Instead, the motion would be switched to PWM signal to make electronic equipment read signal. After receiving the signal, electronic equipment would switch it to angle and angular velocity again, then deliver data into flight control system to calculate them. The linear operation formula expresses as (1).

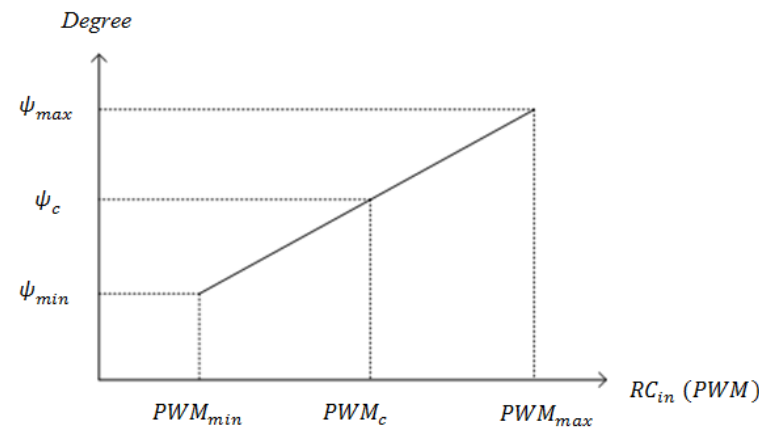

Figure 6 The transformation of PWM and pitch angle

$$
\begin{gathered}
\frac{\psi_{c}-\psi_{\min }}{P W M_{c}-P W M_{\min }}=\frac{\psi_{\max }-\psi_{\min }}{P W M_{\max }-P W M_{\min }} \\
\psi_{c}=\psi_{\min }+\left(P W M_{c}-P W M_{\min }\right) \times \frac{\psi_{\max }-\psi_{\min }}{P W M_{\max }-P W M_{\min }}
\end{gathered}
$$

Where $\psi$ : pitch angle

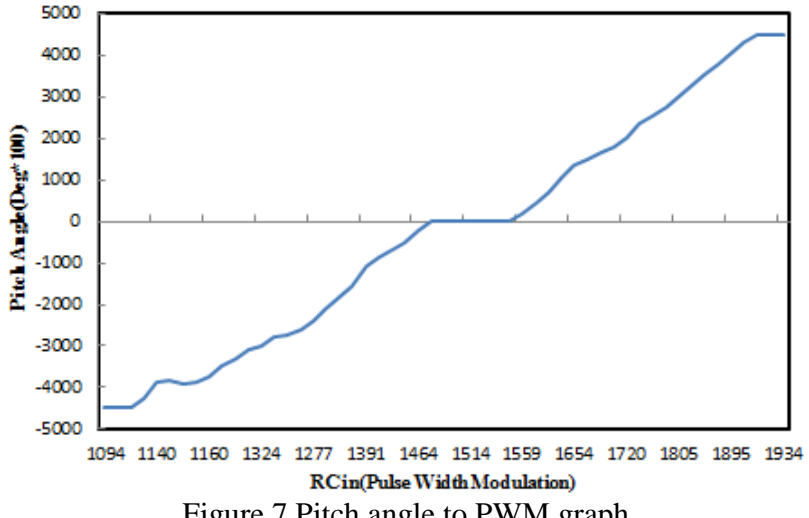

Figure 7 Pitch angle to PWM graph

\subsection{Decision Tree}

Decision tree which classifies data from top to bottom is a data structure. According to the problems, the operation would be expressed as a tree-like graph.

The research utilizes Classification and Regression Tree which is binary division to analyse contiguous and quantitative data. The method is to divide every node into two branches and use TRUE or FALSE as judgement.

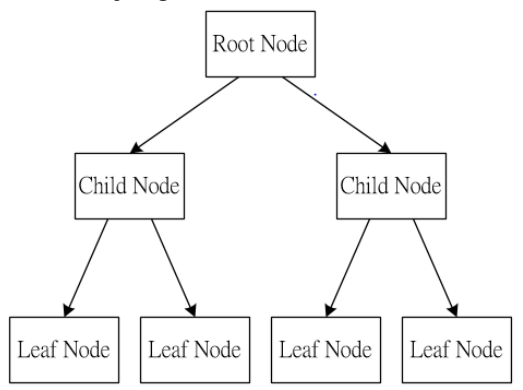

Figure 8 Decision tree

\subsection{The concept of collision avoidance}

The concept of collision avoidance divides into operation control and collision avoidance control which is divided into slow avoidance area and rapid avoidance area in order not to change pitch angle largely. This can make the quadcopter moves smoothly when the quadcopter implements collision avoidance motion. After implementing slow avoidance area at the slow flight condition, the system returns the authority to the operator. In the rapid flight condition, the slow avoidance area could provide initial decelerating effect, while the quadcopter flies fast. Then the system enters rapid avoidance area to provide larger pitch angle to make the quadcopter fly away from the obstacles.

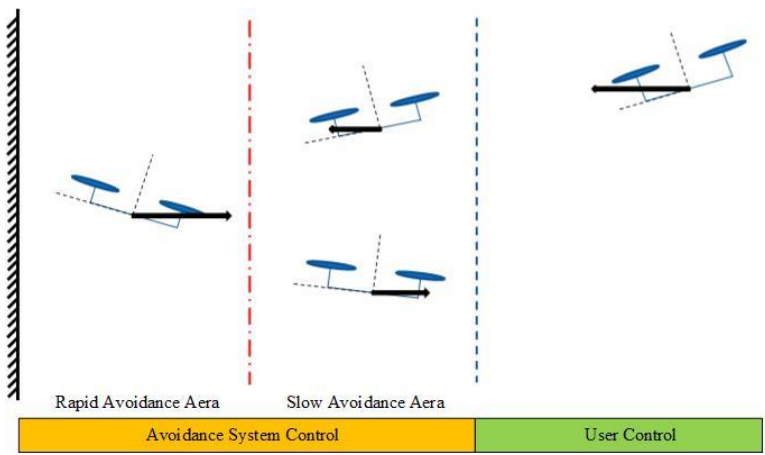

Figure 9 The concept of collision avoidance 


\subsection{Decision Tree of Collision Avoidance}

The quadcopter would implement corresponding collision avoidance motions based on the distance value measured by the ultrasonic range finder. As seen in Figure 10.

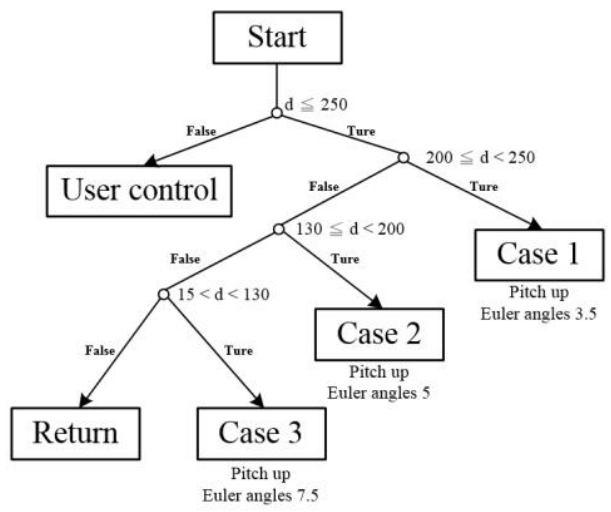

Figure 10 Collision Avoidance Decision Tree

\subsubsection{User control :}

The operator could operate the quadcopter arbitrarily when the distance value is not lower than $250 \mathrm{~cm}$. The collision avoidance system would not interfere the operator.

\subsubsection{Case1:}

When the ultrasonic range finder detects the distance between the quadcopter and the obstacle is in the range of $200 \mathrm{~cm}$ to 250 $\mathrm{cm}$, the collision avoidance system activates. Meanwhile, the authority of the collision avoidance system is higher than the operator and the collision avoidance system will order the flight control board to pitch up to 3.5 degrees to leave the area or achieve the initial deceleration to Case2.

\subsubsection{Case2:}

If the quadcopter continues to approach to the obstacle, and the distance between the quadcopter and the obstacle is in the range of $130 \mathrm{~cm}$ to $200 \mathrm{~cm}$, the collision avoidance system will order the flight control board to pitch up to 5 degree to make quadcopters leave the area, otherwise the control state would transfer to case 3 .

\subsubsection{Case3:}

Rapid avoidance stage is activated when ultrasonic range finder detects the distance between the quadcopter and the obstacle is in the range of $15 \mathrm{~cm}$ to $130 \mathrm{~cm}$, the collision avoidance system will order the flight control board to pitch up to 7.5 degree to make quadcopters leave the area and transfer to case2.

\subsubsection{Return}

Return block means the process would return to the origin of decision tree, then restart the process repeatedly.

The collision avoidance system only restricts the operation of pitch control, however, the authority of control about rolling, yawing and the throttle are still belong to the operator. The operator could control the other parameters according to the situation to make the quadcopter stable.

\section{THE STRUCTURE OF COLLINSION AVOIDANCE SYSTEM}

Integrate the collision avoidance decision tree to the quadcopter and understand its input and output. Operating the quadcopter directly or the feedback distance value which is the decision tree reference is input. Output is the corresponding altitude. Finally the quadcopter follows the corresponding motion and modifies the altitude with $400 \mathrm{~Hz}$ frequency. The flight control board would update the distance value acquired by the ultrasonic range finder with $10 \mathrm{~Hz}$ and implement the collision avoidance system continuously. Meanwhile the operator could check state of the system through the ground data station.

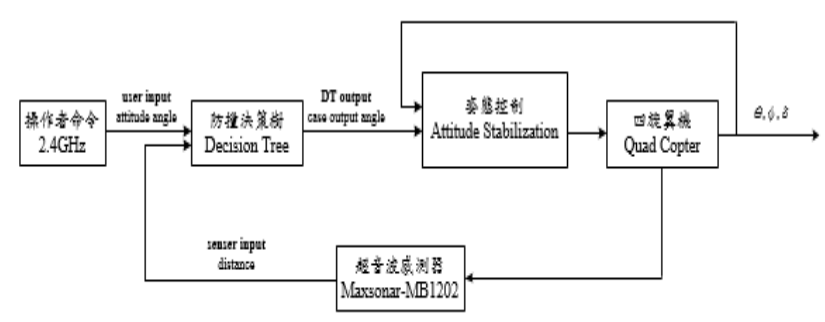

Figure 11 Block of Integrated system graph

\section{FLIGHT TEST AND DISCUSSION}

The test location is outdoor and there is a roller shutter with large area as the obstacle. The purpose of test is to test the effect of collision avoidance with decision tree algorithm. Testing time is $3 \mathrm{~m} 08 \mathrm{~s}$ and dividing the entire flight data in three parts. The first timeline is from $10 \mathrm{~s}$ to $68.3 \mathrm{~s}$. From $68.3 \mathrm{~s}$ to $122.1 \mathrm{~s}$ is the time to adjust the quadcopter on the ground. At last part is to test the authority. Figure 12 and Figure 13 are the flight data from the beginning to the end.

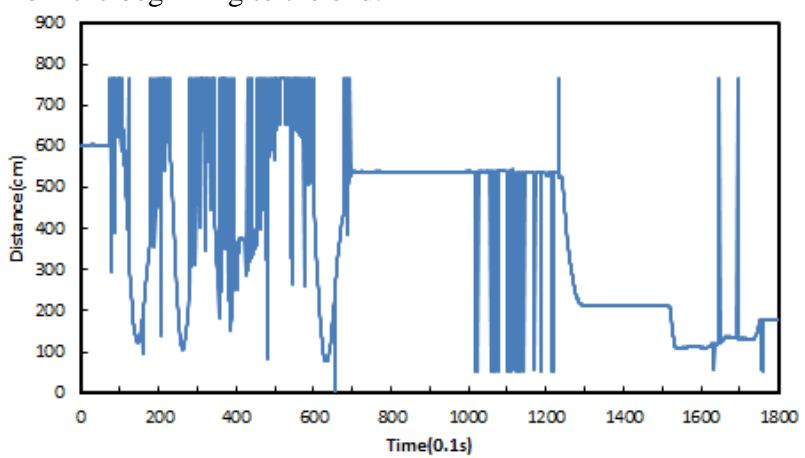

Figure 12 The whole flight distance value

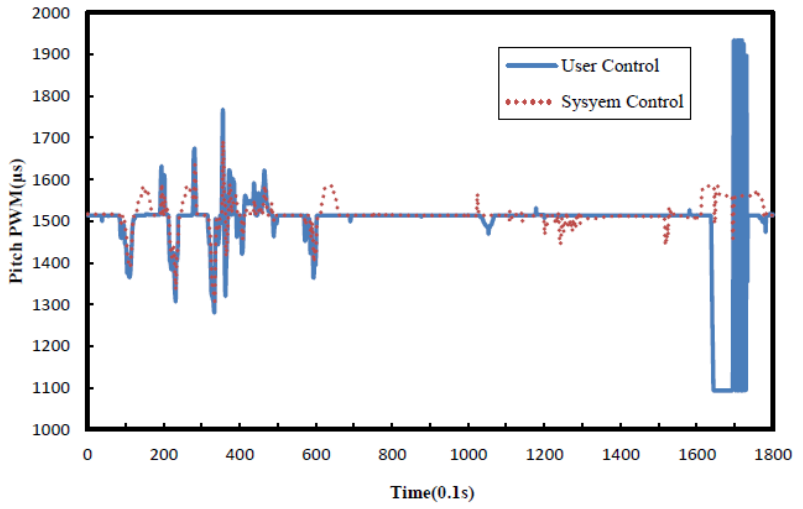

Figure 13 The whole flight pitch angle data 
At $12.9 \mathrm{~s}$, the quadcopter flied into avoidance interval as seen in figure14. The collision avoidance system interfered the operation and made the quadcopter pitch up according to the different distance value. At $16.1 \mathrm{~s}$, the distance value returned within $130 \mathrm{~cm}$. so in Figure 15 and Figure 16, the pitch angle rose deeply.

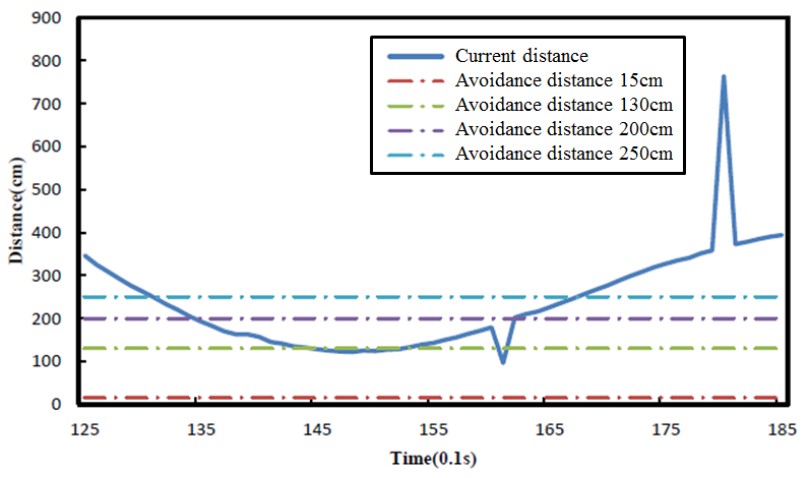

Figure 14 The distance value $-1^{\text {st }}$

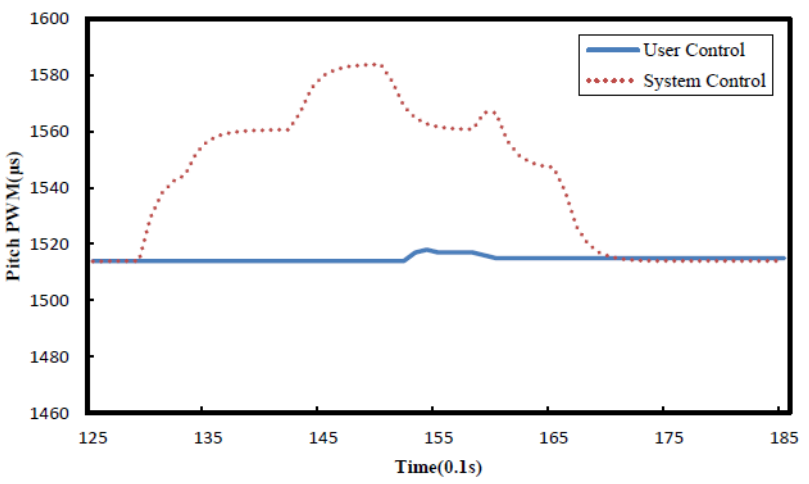

Figure 15 User control and System control

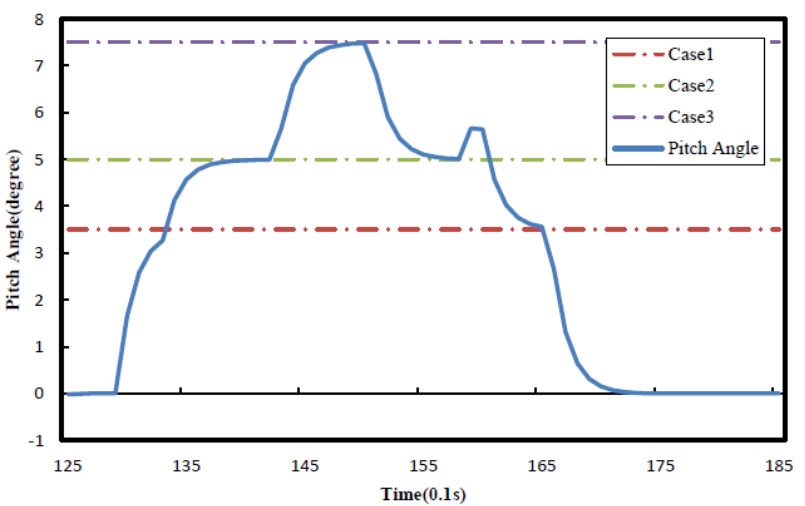

Figure 16 Pitch angle graph- $1^{s t}$

\begin{tabular}{|c|c|}
\hline $\begin{array}{c}\text { Before triggering collision } \\
\text { avoidance velocity }(\mathrm{m} / \mathrm{s})\end{array}$ & 1.52 \\
\hline $\begin{array}{c}\text { Disengaged collision avoidance } \\
\text { velocity }(\mathrm{m} / \mathrm{s})\end{array}$ & 0.91 \\
\hline The minimum distance $(\mathrm{m})$ & 1.21 \\
\hline
\end{tabular}

Table 1 Collision avoidance events and minimum distance $-1^{\text {st }}$

The second collision avoidance decision tree data are from $24.5 \mathrm{~s}$ to $29.5 \mathrm{~s}$ illustrated in Figure 17, Figure 18, and Figure 19. Before the system triggered collision avoidance, the operator pushed the remote controller to make the quadcopter test collision avoidance with 1.9 meter per second. However, at $28.1 \mathrm{~s}$, the system detected that the distance was over $2.5 \mathrm{~m}$ between the obstacle. It means that disengages the system control. The interest thing is that after disengaging the collision avoidance loops, the system only implements decision tree and processes case 2 rather than case 1 . Then the quadcopter's pitch control matched the operator's manipulation again. It was verified that if it was not in the area, the authority of operation would return to the operator.

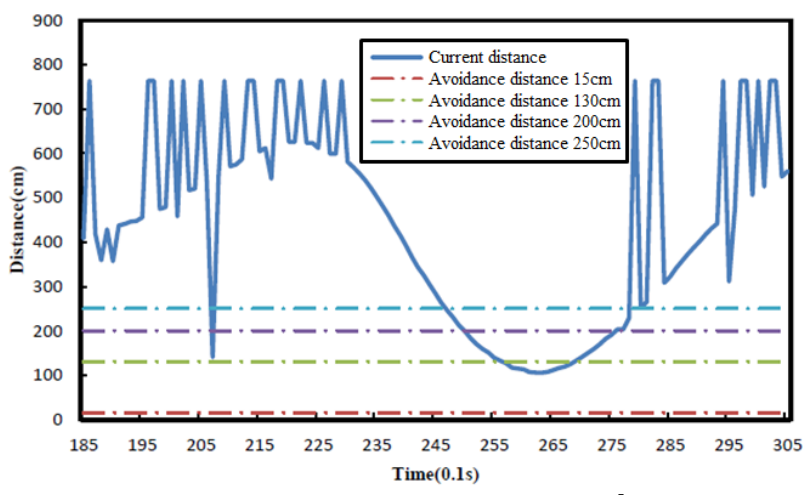

Figure 17 The distance value $-2^{\text {nd }}$

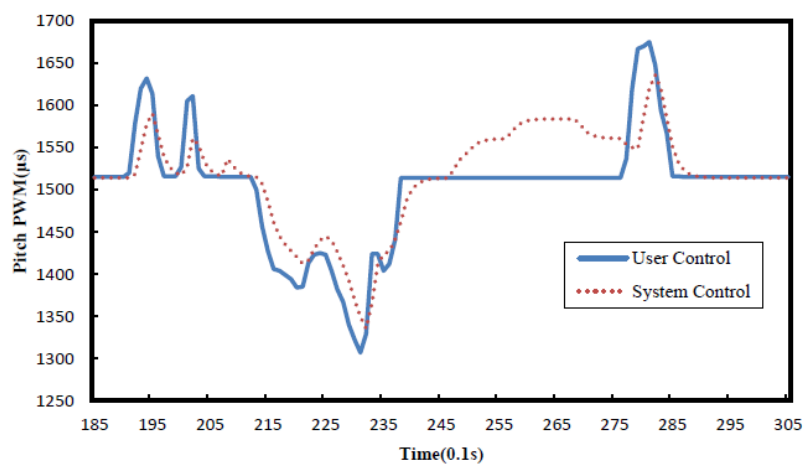

Figure 18 User control and System control- $2^{\text {nd }}$

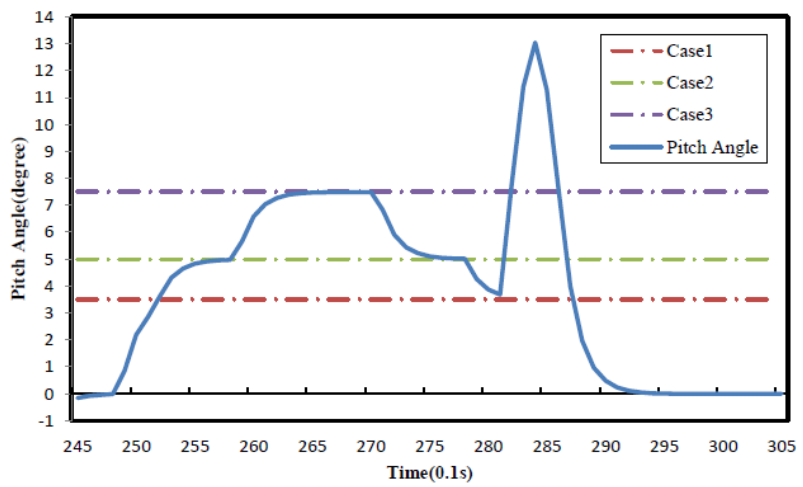

Figure 19 Pitch angle graph $-2^{\text {nd }}$

\begin{tabular}{|c|c|}
\hline $\begin{array}{c}\text { Before triggering collision } \\
\text { avoidance velocity }(\mathrm{m} / \mathrm{s})(\mathrm{m} / \mathrm{s})\end{array}$ & 1.92 \\
\hline $\begin{array}{c}\text { Disengaged collision avoidance } \\
\text { velocity }(\mathrm{m} / \mathrm{s})\end{array}$ & 0.96 \\
\hline The minimum distance $(\mathrm{m})$ & 1.05 \\
\hline
\end{tabular}

Table 2 Collision avoidance events and minimum distance $2^{\text {nd }}$

In the last part, a man took the quadcopter to get close to the obstacle and to observe the variation of pitch, the authority 
between the operator and the system shown in Figure 20, Figure 21 and Figure 22. No matter how the operator controls, the inputs will be ignored when the collision avoidance system is activated until it disengages the area. Nevertheless, within the area the system only constrains pitch control. The operator could control such as rolling, yawing, and throttle.

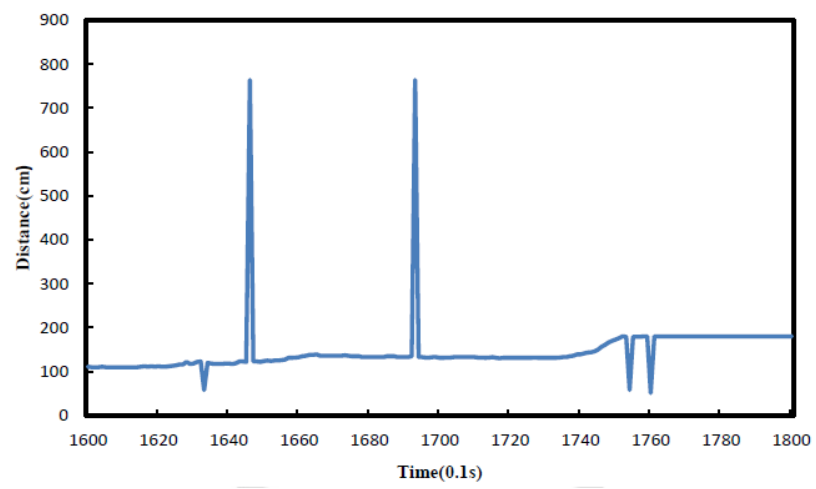

Figure 20 The distance value -

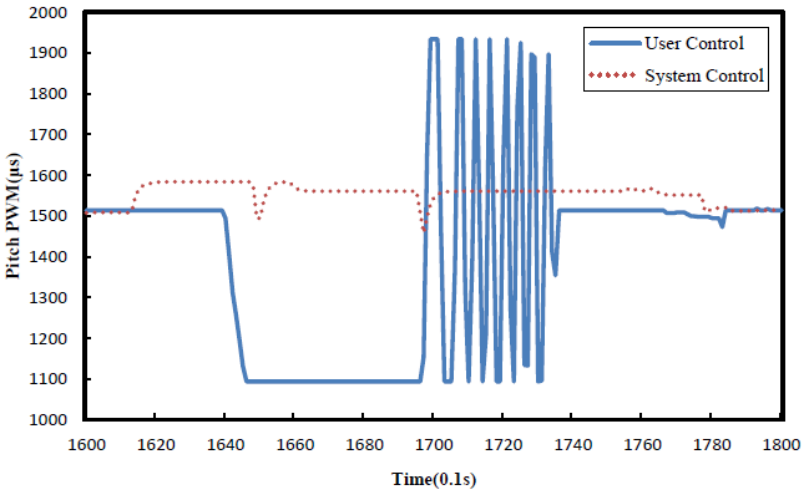

Figure 21 User control and System control-

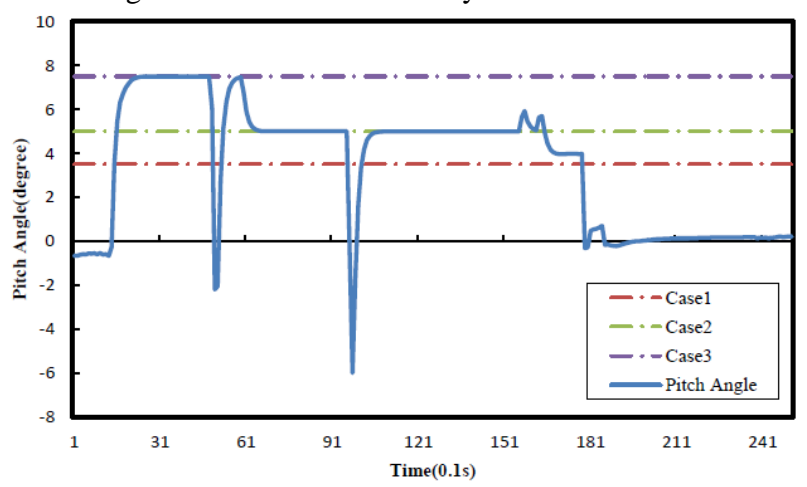

Figure 22 Pitch angle graph -

\section{CONCLUTION}

The research is to develop the collision avoidance system used for the quadcopter based on Pixhawk flight control board embedded with the collision avoidance algorithm, sensors and the data transmission program in object orientation structure and to construct ground data station to analyse data. Obtain the relative distance to the front obstacle by the ultrasonic range finder. Within the collision avoidance area, the system decides the corresponding altitude to the quadcopter by the decision tree collision avoidance algorithm. The experiment verifies that the quadcopter can avoid the obstacle effectively with approximately the speed of 1.9 meter per second. But the system can only control the pitch angle. If the operator corrects the throttle inappropriately, the quadcopter would drop height a little bit during the avoidance motion.

\section{REFERENCES}

Chee, K.Y., Zhong, Z.W. 2013, "Control, Navigation and Collision Avoidance for an Unmanned Aerial Vehicle," Sensors and Actuators A:Physical, vol190, pp.66-76,.

Sun, H., Hu, X., 2017. "Attribute Selection for Decision Tree Learning with Class Constraint," Chemometrics and Intelligent Laboratory System, vol.163, pp.16-23.

Tripathi, A. K. Raja, R. G. Padhi, R. 2014, "Reactive Collision Avoidance of UAVs with Stereovision Camera Sensors using UKF,” IFAC Proceedings Volumes, vol.47, pp.1119-1125.

Westreich, D., Lessler J., Funk, M. J., 2010, "Propensity Score Estimation: Neural Networks, Support Vector Machines, Decision Trees (CART), and Meta-Classifiers as Alternatives to Logistic Regression,” Journal of Clinical Epidemiology, vol.63, pp.826-833.

Wu, B.-S., 2015, "The Design and Verification of CollisionAvoidance for Quad Copter," MS Thesis of Department of Aeronautics and Astronautics, National Cheng Kung University, Taiwan.

Xiao, C.-R., 2009, “Application of Fuzzy Decision Tree Control Method in Developing a Collision-Avoidance System on a Robot Car," MS Thesis of Department of Aeronautics and Astronautics, National Cheng Kung University, Taiwan. 